\title{
Developing inclusive primary care for trans, gender-diverse and nonbinary people
}

\author{
Riki Lane PhD
}

- Cite as: CMAJ 2019 January 21;191:E61-2. doi: 10.1503/cmaj.190011

See related articles at www.cmaj.ca/lookup/doi/10.1503/cmaj.180839, www.cmaj.ca/lookup/doi/10.1503/cmaj.180672, www.cmaj.ca/ lookup/doi/10.1503/cmaj.181047, and www.cmaj.ca/lookup/doi/10.1503/cmaj.180954

W

orldwide, meeting the health needs of trans, genderdiverse and nonbinary (TGDNB) people is shifting toward primary care. Presentations to health care providers regarding gender transition are also increasing rapidly. Therefore, all primary care providers need familiarity with TGDNB treatment guidelines and inclusive practice, and more primary care clinicians are gaining the experience and confidence to manage gender assessments and transitions.

Four articles collected in this issue of CMAJ raise a number of issues that health care providers should be aware of when working with this population. A large survey by Pinto and colleagues ${ }^{1}$ considers the acceptability to clients of including options beyond male and female in clinical records systems; Bonifacio and colleagues ${ }^{2}$ discuss the management of gender dysphoria in adolescents by primary care practitioners, providing extensive background and guidance on affirmative care; Beswick and colleagues' case report ${ }^{3}$ offers succinct advice on improving processes for cervical cancer screening in trans men; and Lam and Abramovich ${ }^{4}$ concisely outline key points for achieving inclusive practice for TGDNB people.

Terminology is challenging in this fast-moving field; "umbrella" terms vary across time and nations, including transgender, trans, trans ${ }^{\star}$, trans and gender-diverse, trans and gender-nonconforming. I use "TGDNB," as advised by our consumer advisory panel at the Monash Health Gender Clinic in Australia. In the Canadian context, Pinto and colleagues ${ }^{1}$ include "2-spirit" for Indigenous TGDNB people in their broader term: lesbian, gay, bisexual, transgender, transsexual, queer, questioning and 2-spirit (LGBTQ2S).

A key shift is from a binary notion of gender as only male or female, with some people moving from one to the other, to seeing gender identity as a spectrum or galaxy. Readers can see some of these shifts in language between the survey questions used in Pinto and colleagues' study, conducted from 2013 to $2016,{ }^{1}$ to the terminology used in Bonifacio and colleagues' more recent review of the care of adolescents who identify as TGDNB. ${ }^{2}$ The use of "female to male" ("FTM") by Beswick and colleagues, ${ }^{3}$ while still used as an identifier by some trans men, has recently fallen from favour, especially with increasing nonbinary gender

\section{KEY POINTS}

- Primary care providers are playing an increasing role in health care for trans, gender-diverse and nonbinary (TGDNB) people.

- At the organizational level, amending clinical record systems can make them TGDNB inclusive.

- At the cultural level, staff training can develop affirmative care practices, especially respectful use of language.

- At the clinical level, staff can be aware of particular practical issues, including managing co-occurring conditions while patients are waiting for gender assessment; gaining experience in undertaking gender assessments; correcting erroneous assumptions about the need for cancer screening; and discussing fertility preservation before transition.

identifications, such as bigender (alternating between female and male) or neither male nor female. "Female to male" now usually describes 1 binary process of transition among a range of possibilities, rather than describing people.

Trans, gender-diverse and nonbinary people often face difficulties in having their gender identity recognized in clinical records systems. Pinto and colleagues' mixed-methods study ${ }^{1}$ provides useful guidance. The survey portion included TGDNB people at higher rates than usual in clinical samples: $1.02 \%$ had a diagnosis for gender dysphoria, and $1.22 \%$ had no diagnosis, but their gender identity differed from clinical records. However, interpreting the interview portion of the study is somewhat difficult, given a skewed gender ratio (18/27 female) and limited information on gender identity and sexual orientation. However, most participants welcomed the variety of gender-identity options available, although some respondents were discomfited. The study findings support several key recommendations for primary care practices and researchers. At the organizational level, amending clinical practice record systems can make them TGDNB inclusive. A two-stage process for gender identity is best, with separate questions about, first, sex assigned at birth, and second, current gender identity, followed by a free-text option for clarification. Furthermore, working with LGBTQ2S communities to 
standardize questions is important.Training staff will also help with building an inclusive clinical culture for TGDNB and other LGBTQ2S people.

Bonifacio and colleagues ${ }^{2}$ deliver well-researched advice on issues facing primary clinicians in the management of TGDNB adolescents, outlining the stages of care from affirming the person at initial presentation, considering a diagnosis, seeking expert help if required, supporting young people through social and medical gender transition, and highlighting some gaps in knowledge and controversies. Although experienced non-mental health clinicians often conduct gender assessments, inexperienced ones may need to refer out. In the latter case, the authors advise managing cooccurring conditions such as anxiety while awaiting gender assessment. The article focuses on adolescents, but much material is relevant to children, such as the timing of prescribing puberty blockers. To the resources list in the Bonifacio article, ${ }^{2}$ I suggest 1 addition: the 2017 "Australian standards of care and treatment guidelines for transgender and gender diverse children and adolescents, " 5,6 recently praised by The Lancet. $^{7}$

Beswick and colleagues' case report ${ }^{3}$ is a cautionary tale of the dangers of making assumptions about gender as binary, and about the sexual practices of TGDNB people. A clinician thought that only penile penetrative intercourse could cause transmission of human papillomavirus, ignoring oral or digital transmission routes. Thus, a trans man was incorrectly advised that cervical cancer screening was unnecessary. Similar issues may arise with cancer screening among other TGDNB people. A recent study shows reduced screening rates for prostate cancer among gender-nonconforming people and for colorectal cancer among trans women. ${ }^{8}$ Beswick and colleagues ${ }^{3}$ highlight concerns for trans men when undergoing cervical examinations, and a low participation rate in cervical screening, owing to both bodily discomfort and previous experiences of stigmatization and discrimination from health providers. Pointing to the excellent 2015 guidance article by Potter and colleagues, ${ }^{9}$ which delivers clear, concise advice on procedures, Beswick and colleagues highlight the need for primary care providers to improve the experience of and access to care for TGDNB people.

The short but useful article by Lam and Abramovich ${ }^{4}$ includes important points that are alluded to in other articles in the issue. They note that estimates regarding the TGDNB population keep rising, as do presentations to health care services. Primary care clinicians play increasing roles in the management of the health care of TGDNB people and guidelines are available to support prac- tice. Respecting pronouns, names and gender markers requested by TGDNB clients is essential. TGDNB-sensitive approaches can improve cancer screening. Discussion on fertility preservation is required before hormonal treatments for gender transition.

Trans, gender-diverse and nonbinary people are at high risk for suicide, especially when access to medical transition services is limited, ${ }^{10,11}$ so prolonging a wait for gender assessment may be harmful. It is important to refer out for co-occurring conditions (especially mental health conditions), speech therapy and hair removal.

In conclusion, the articles collected in this issue should be key reading for primary care providers, in their endeavours to develop inclusive and affirmative care for TGDNB people.

\section{References}

1. Pinto AD, Aratangy $T$, Abramovich A, et al. Routine collection of sexual orientation and gender identity data: a mixed-methods study. CMAJ 2019;191:E63-8.

2. Bonifacio JH, Palmert M, Stadelman K. Management of gender dysphoria in adolescents in primary care. CMAJ 2019;191:E69-75.

3. Beswick A, Corkum M, D'Souza D. Locally advanced cervical cancer in a transgender man. CMAJ 2019;191:E76-8.

4. Lam JSH, Abramovich A. Transgender-inclusive care. CMAJ 2019;191:E79.

5. Telfer MM, Tollit MA, Pace CC, et al. Australian standards of care and treatment guidelines for transgender and gender diverse children and adolescents. Med J Aust 2018;209:132-6.

6. Telfer M, Tollit M, Pace CC, et al. Australian Standards of Care and Treatment Guidelines for trans and gender diverse children and adolescents. Melbourne (Australia): The Royal Children's Hospital; 2017. Available: www.rch.org.au/adolescent -medicine/gender-service/ (accessed 2018 Dec. 20).

7. Gender-affirming care needed for transgender children. Lancet 2018;391:2576.

8. Tabaac AR, Sutter ME, Wall CSJ, et al. Gender identity disparities in cancer screening behaviors. Am J Prev Med 2018;54:385-93.

9. Potter J, Peitzmeier SM, Bernstein I, et al. Cervical cancer screening for patients on the female-to-male spectrum: a narrative review and guide for clinicians. J Gen Intern Med 2015;30:1857-64.

10. Bauer GR, Scheim Al, Pyne J, et al. Intervenable factors associated with suicide risk in transgender persons: a respondent driven sampling study in Ontario, Canada. BMC Public Health 2015;15:525

11. Smith AJ, Hallum-Montes R, Nevin K, et al. Determinants of transgender individuals' well-being, mental health, and suicidality in a rural state. Rural Mental Health 2018;42:116-32.

\section{Competing interests: None declared.}

This article was solicited and has not been peer reviewed.

Affiliations: Southern Academic Primary Care Research Unit, Department of General Practice, Monash University, Notting Hill; Gender Clinic, Monash Health, Hampton East, Australia

Correspondence to: Riki Lane, riki.lane@monash.edu 